Military Technical College

Kobry El-Kobbah Cairo, Egypt

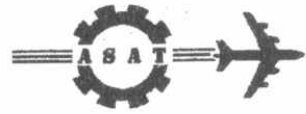

$10^{\text {th }}$ International Conference

On Aerospace Sciences\&

Aviation Technology

\title{
ROCKET ASSISTED PROJECTILES; A COMPUTATIONAL ANALYSIS OF TRAJECTORY
}

\author{
Sarhan, A. M. ${ }^{*}$, El-Senbawi, M. A.** and Nafea A.***
}

\section{ABSTRACT}

A versatile computer program is coded, using MATLAB (SIMULINK), to compute the trajectory elements of the rocket assisted projectiles (range flight velocity and inclination angle). The inputs of the code, are the projectile parameters (mass, caliber, muzzle velocity, drag coefficient and firing angle) and the assisting rocket parameters (mass, time of function, delay time and propellant specific impulse). The code is used to study different design factors affecting the range of the rocket assisted projectiles. It is found that the range can be significantly extended by using an assisting rocket. The range extension increases by using greater propellant mass, smaller delay time and smaller time of function. The angle of the maximum range is a function of the propellant to total projectile mass ratio. In the investigated case, this angle does not depend on the delay time or the time of function.

\section{List of symbols}

$C_{D} \quad$ drag coefficient.

D drag.

$F, \sum \vec{F}_{\text {ex }} \quad$ thrust, and Sum of the external forces, respectively.

g gravity acceleration,

h height.

Isp specific impulse

ITO total impulse.

$M \quad$ Mach number.

$m, m_{P}, m_{T}$ mass, propellant mass, total mass, respectively.

$\dot{m} \quad$ mass flow rate of the exhausted gases.

p pressure.

$\mathrm{S}_{\pi} \quad$ reference area ( projectile cross section area).

$T^{n}$ temperature.

$t, t_{k} \quad$ time, assisting rocket time of function, respectively.

$\mathrm{V}, \mathrm{u}, \mathrm{v} \quad$ velocity, horizontal and vertical velocity components, respectively.

$X, Y \quad h o r i z o n t a l$ and vertical axes of inertial frame of reference, respectively.

$\alpha \quad$ inclination angle of the projectile.

$\rho \quad$ air density.

* Professor, Egyptian armed forces.

** Assoc. Professor, Egyptian armed forces.

*** Ass. Prof., Egyptian armed forces. 


\subsection{INTRDUCTION}

$R_{\text {ange extension of artillery has always been and will continue to be an }}$ essential goal and crucial demand of conventional and non-conventional artillery designers. To achieve this objective, attention has been focused on increasing the muzzle velocity of projectile and improving its aerodynamic shape and ballistic performance. See Plate 1.

Projectile muzzle velocity can be increased by using greater propellant mass or energy and/or longer length of gun barrel. The resulting increase in recoil energy not only affects adversely the stability of the gun but also necessitates major changes and efficient design of most of gun systems and subsystems. Undesirable heavier gun with lower maneuverability is expected.

Reduction of the aerodynamic drag of the fore body by improving the aerodynamic shape of projectile via elimination of the cylindrical central part and incorporating a longer full ogive body reduces the head resistance (wave drag) by about $30 \%$, plate 1-b. This solution, however, leads to less stable projectile along trajectory and a subsequent higher dispersion.

Reduction of the base drag acting on projectile passively via Boat Tailing (BT), plate 1-C, [1] and/or Base Bleed (BB),[2\&3], though successful and encouraging, but is limited to 20 up to $25 \%$ extension in range.

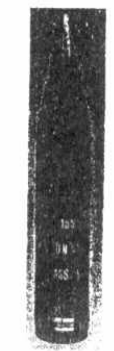

a- standard

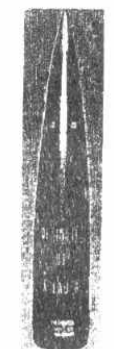

b- fore body modified

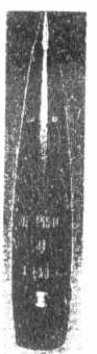

c- boat tailed

Plate 1 Aerodynamic shape modification of $155 \mathrm{~mm}$ projectile

In the past decade, the use of Rocket Assisted Projectile (RAP) has impacted on range extension of conventional and non-conventional artillery. It has the advantage over the increase of muzzle velocity technique in that RAP can be fired from already existing guns. With the potential of about $46 \%$ range extension, RAP meets the ambition of reaching a range over $80 \mathrm{~km}$ with the future $21^{\text {st }}$ Century $155 \mathrm{~mm}$ Field Howitzer and makes it within reach, [4\&5].

The desire and need to resolve some ambiguities concerning range extension via RAP suggest the need for a more computational analysis of RAP trajectory. The primary goal is to get clearer picture and to find answers to questions as to: what is 
the required rocket mass to gain a specified range extension?, what is the optimum duration of the assisting rocket function?, what is the optimum delay time of starting the assisting rocket? and what is the firing angle which gives the maximum range?

The present work is a computational analysis of the trajectory of the Rocket Assisted Projectile, RAP, based on experimentally determined drag coefficient regime of an available $155 \mathrm{~mm}$ projectile. The programmed code can also be implemented to study any other projectile provided that its mass, muzzle velocity and drag coefficient are known.

\subsection{THEORETICAL ANALYSIS}

When a projectile is fired from a gun barrel; the produced high pressure and high temperature burnt gases accelerate the projectile during its travel down the barrel. The muzzle energy of projectile is directly proportional with the area under the pressure travel curve. The muzzle velocity depends mainly on the mass and energy of the propellant used and the length of the barrel. The maximum range of the projectile is a function of the square of the gained muzzle velocity. In the case that the muzzle velocity can not be increased any more inside the barrel; the velocity can be increased further outside the barrel by the help of an assisting rocket.

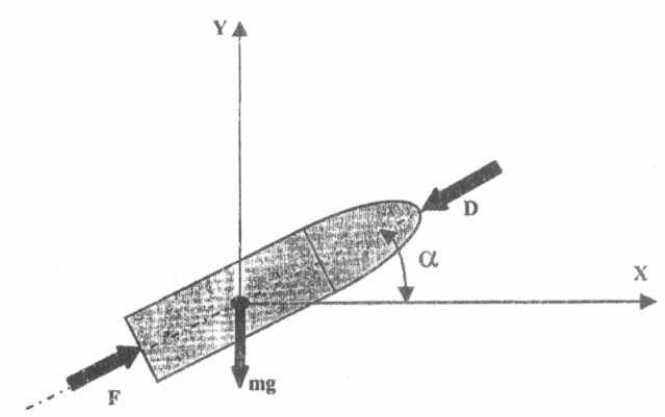

Fig. 1 Forces acting on the rocket assisted projectile during flight

The equation of motion of the projectile during its flight is written in the vector form, neglecting the lift force as:

$$
m(t) \frac{d \vec{V}}{d t}=\sum \vec{F}_{e x}
$$

where $m(t)$ is the projectile mass as function of time, $V$ is the projectile velocity and $\sum \vec{F}_{\text {ex }}$ is the sum of the external forces which include, the thrust, $\vec{F}$, the drag force, $\vec{D}$, and the gravity force, $m(t) \vec{g}$. 
Resolution of equation (1) into the $\mathrm{X}$ and $\mathrm{Y}$ directions gives:

$$
\begin{aligned}
& m(t) \frac{d u}{d t}=F \cos \alpha-D \cos \alpha \\
& m(t) \frac{d v}{d t}=F \sin \alpha-D \sin \alpha-m(t) g
\end{aligned}
$$

where $u, v, F, D, m, t, \alpha$, are velocity components in $X$ and $Y$ directions, thrust, drag, mass, time and inclination angle of the projectile respectively.

The thrust is expressed as:

$$
\mathrm{F}=\dot{\mathrm{m}} \mathrm{I}_{\mathrm{SP}}
$$

where $\dot{m}$ and $I_{s p}$ are the mass flow rate of the exhausted gases from the assisting rocket and its specific impulse, respectively.

The total impulse, $I_{\text {TOT, }}$, of the rocket, over its time of function, $t_{k}$, is expressed as:

$$
\mathrm{I}_{\text {TOT }}=\int_{0}^{\mathrm{t}_{\mathrm{k}}} \mathrm{F} d \mathrm{~d}
$$

The drag is expressed as:

$$
D=1 / 2 \rho V^{2} C_{D} S_{\pi}
$$

where, $\rho, V, C_{D}$ and $S_{\pi}$ are air density, projectile velocity, drag coefficient, and the reference area ( cross section area of the projectile).

The air parameters are expressed in Table 1 as functions of the altitude as [6],

Table 1 Temperature and pressure as functions of altitude

\begin{tabular}{|c|c|c|}
\hline Altitude, $h,[\mathrm{~m}]$ & Temperature, $\mathrm{T},\left[{ }^{\circ} \mathrm{C}\right]$ & Pressure, $\mathrm{p},[\mathrm{kPa}]$ \\
\hline $\begin{array}{c}\mathrm{h} \geq 25000 \mathrm{~m} \\
\text { (Upper Stratosphere) }\end{array}$ & $\mathrm{T}=-131.21+0.0299 \mathrm{~h}$ & $\mathrm{p}=2.488\left[\frac{\mathrm{T}+273.1}{216} \cdot\right]^{-11.388}$ \\
\hline $\begin{array}{c}11000<\mathrm{h} \leq 2500 \mathrm{~m} \\
\text { (Lower Stratosphere) }\end{array}$ & $\mathrm{T}=-56.46$ & $\mathrm{p}=22.65 \mathrm{e}^{(1.73-0.00157 \mathrm{~h})}$ \\
\hline $\begin{array}{c}\mathrm{h}<11000 \mathrm{~m} \\
\text { (Troposphere) }\end{array}$ & $\mathrm{T}=15.04-0.0649 \mathrm{~h}$ & $\mathrm{p}=101.29\left[\frac{\mathrm{T}+273.1}{288.08}\right]^{5.256}$ \\
\hline
\end{tabular}

For all altitudes, the density of the air is

$$
\rho=\frac{p}{(28699)(T+273.1)}
$$




\subsection{COMPUTATIONAL ANALYSIS}

In the present work, the case of $155 \mathrm{~mm}$ Howitzer is implemented to investigate the effect of the main design parameters on the range of the rocket assisting projectiles. These parameters are:

- The ratio of assisting rocket propellant mass to the total projectile mass.

- The delay time of starting the assisting rocket.

- The time of function of the assisting rocket.

- The firing angle which gives the maximum range for different ratios of assisting rocket propellant mass to the total projectile mass, delay times of starting the assisting rocket. and times of function of the assisting rocket.

The mass of projectile implemented for this study is $42 \mathrm{~kg}$, and its muzzle veløcity is $684 \mathrm{~m} / \mathrm{s}$. A portion of the original mass of the projectile is replaced by the mass of the assisting rocket, so that the mass of the rocket assisted projectile is kept the same as the original projectile mass. Consequently, the muzzle velocity is kept unchanged.

The shape and dimension of the rocket assisted projectile are also kept identical with that of the original projectile.

Experimentally determined drag coefficient of a similar spin stabilized projectile is implemented, [7], to compute the trajectory of the rocket assisted projectile. The drag coefficient of the projectile is expressed as a function of the Mach number, M, as:

- The subsonic regime, $\quad 0.7<M<0.9$

$$
C_{D}=\frac{0.278}{\sqrt{1-M^{2}}}
$$

- The transonic regime, $\quad 0.9<\mathrm{M}<1.2$

$$
C_{D}=M(8.267-4.087 M)-3.53
$$

- The supersonic regime, $1.2<\mathrm{M}<1.5$

$$
C_{D}=\frac{0.375}{\sqrt{M^{2}-1}}-0.07
$$

The Mach number, $\mathbf{M}$, and the air density, $\rho$, are computed for different altitudes, using Table 1 and equation (7). The specific impulse, $I_{s p}$, of the propellant used is chosen to be $2100 \mathrm{~m} / \mathrm{s}$.

The equation of motion of the rocket assisted projectile is computed using the SIMULINK module. This program can solve for the projectile range and its trajectory elements (velocity, $v$, and inclination angle, $\alpha$ ) versus time or horizontal distance, $\mathrm{x}$. To check the accuracy of the program, a run is made to compute the range of the original projectile with no assisting rocket. The run gave a range of $18.159 \mathrm{~km}$, while the real range of this projectile is $18.09 \mathrm{~km}$. The angle which gives the maximum range is $46^{\circ}$ for both computed and real case. This confirms the accuracy of the 
program. The plot of the computed trajectory is presented in Fig. 2. The computed velocity and inclination angle versus time of flight are presented in Fig. 3.

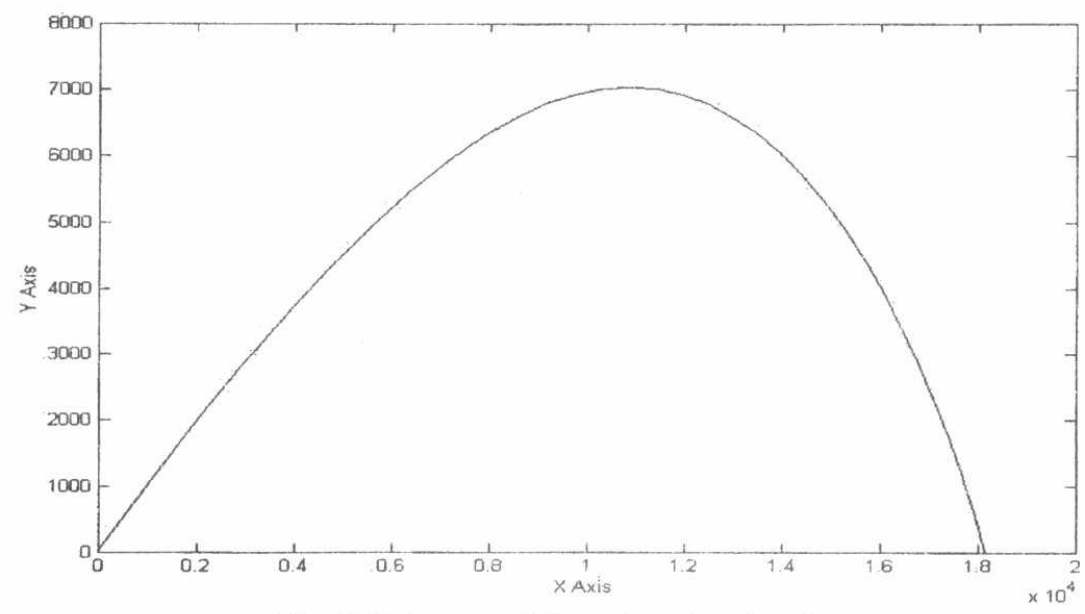

Fig. 2 Trajectory of the original projectile

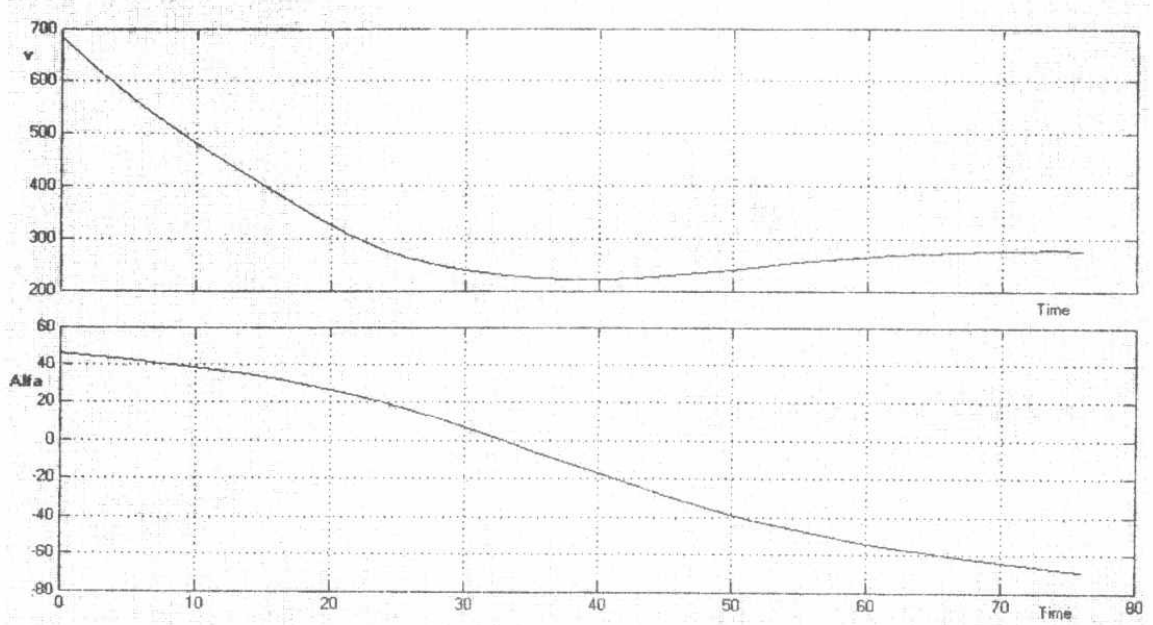

Fig. 3 Velocity and inclination angle of the original projectile versus time of flight

\subsection{Effect of the Ratio of Assisting Rocket Propellant Mass to the Total Projectile Mass}

To investigate the effect of the ratio of assisting rocket propellant mass to the total projectile mass, ratios of $(0$ to $10 \%)$ are examined for a time of function of the assisting rocket; $t_{k}=0.5 \mathrm{~s}$. The assisting rocket is ignited after $1 \mathrm{~s}$ from firing. The computed maximum range for each case is plotted in Fig. 4. The Fig. shows that the range is increased by the increasing the ratio of assisting rocket propellant mass to the total projectile mass. 


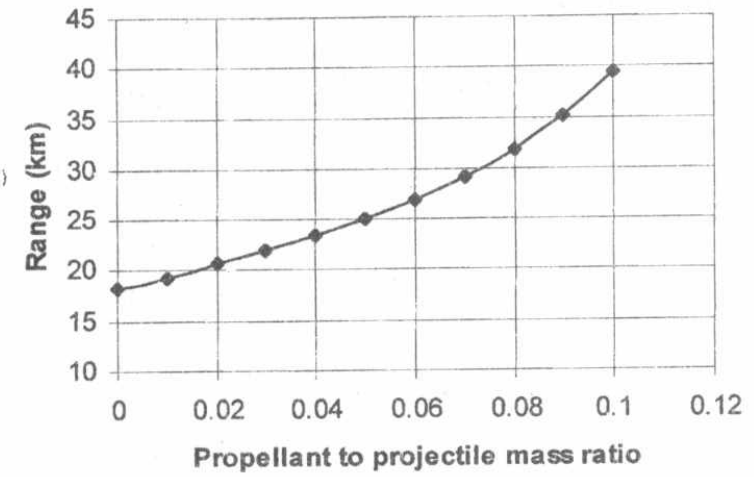

Fig. 4 Dependence of maximum range of the rocket assisted projectile on the ratio of propellant mass to the total projectile mass,

(for time of function of the assisting rocket $=0.5 \mathrm{~s}$, delay time $=1.0 \mathrm{~s}$ )

The program is also used to compute the firing table (range versus firing angle) for mass ratios of $(1,3$ and 5$) \%$ compared to the case of no assisting rocket. The time of function of the assisting rocket is $0.5 \mathrm{~s}$, and its delay time is $1.0 \mathrm{~s}$. The obtained data, presented in Fig. 5, show that for all firing angles the range is increased by the increase of the propellant to total projectile mass ratio.

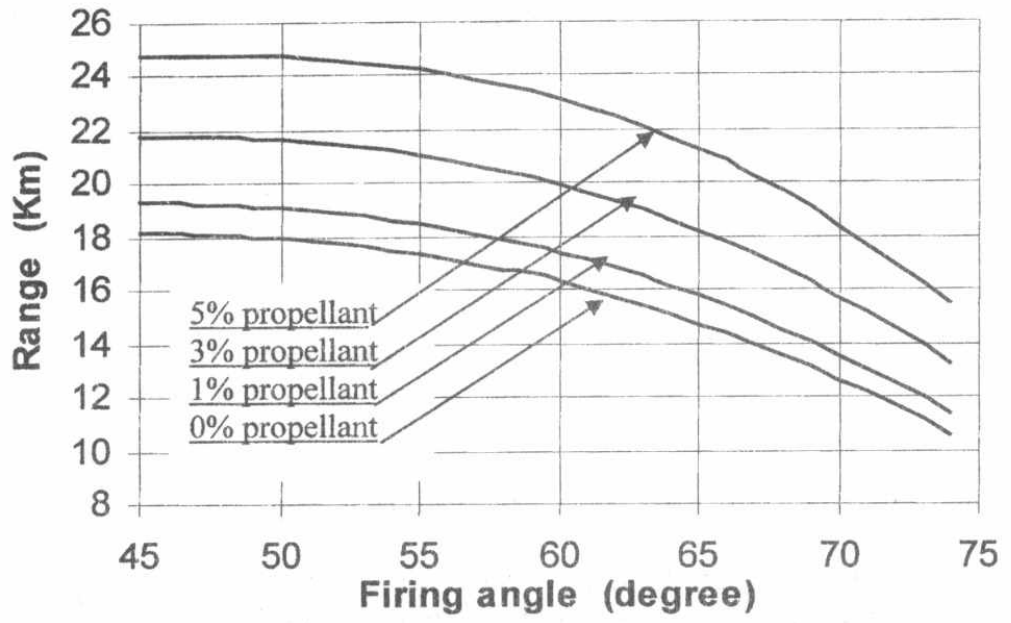

Fig. 5. Range of the projectile versus firing angle for ( propellant masses of $(0,1,3 \& 5) \%$ of the projectile total mass, time of function of the assisting rocket $=0.5 \mathrm{~s}$, delay time $=1.0 \mathrm{~s}$ ) 


\subsection{Delay Time of Starting the Assisting Rocket}

The case of $m_{\rho} / m_{T}=0.05$ is implemented to investigate the effect of the time of function of the assisting rocket on the range of the projectile. The studied times of function are $(0.5,1,2$ and $5 \mathrm{~s})$ and the firing angle is $48^{\circ}$.

Computed data of the range versus delay time is plotted in Fig. 6 . The plot shows that for all investigated times of function of the assisting rocket; the maximum range extension is obtained for zero delay time. The range extension is linearly decreasing with the increase of the delay time in the first one third of the flight period. In the second one third of the flight period; range extension is very small. In the last one third of the flight period; the range does not extend but rather it decreases. Consequently, the delay time of starting the assisting rocket should be as short as possible. It can be concluded that the delay time should be chosen as the minimum delay time which insures the safety of the gunner. The choice of the safe delay time depends on the plume length of the rocket exhausted gases. Consequently, it depends on the thrust of the assisting rocket and the muzzle velocity of the projectile.

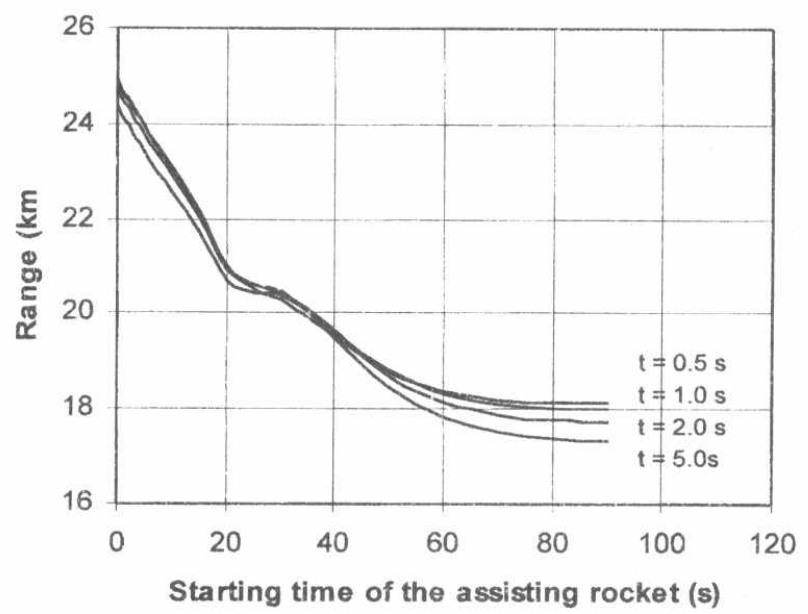

Fig. 6 Effect of the delay time of starting the assisting rocket on the range for (time of function 0.5, 1.0, $2.0 \& 5.0 \mathrm{~s}$. Firing angle $=48^{\circ}$ )

\subsection{Time of Function of The Assisting Rocket}

Fig. 6 shows that the range increases very slightly by the decrease of the assisting rocker time of function. This very weak dependence shows that the range variation (axial dispersion) is small with the variation of the time of function. In fact, the variation of the time of function may result from propellant sensitivity to temperature variation or the production tolerances and inaccuracy.

As an illustrative example the program is used to plot the trajectory, Fig. 7 and velocity and inclination angle, Fig. 8 of a rocket assisted projectile of a ratio of 
propellant to projectile mass $=3 \%$, rocket time of function $=1.0 \mathrm{~s}$, delay time $=2.0 \mathrm{~s}$ and firing angle $=47^{\circ}$

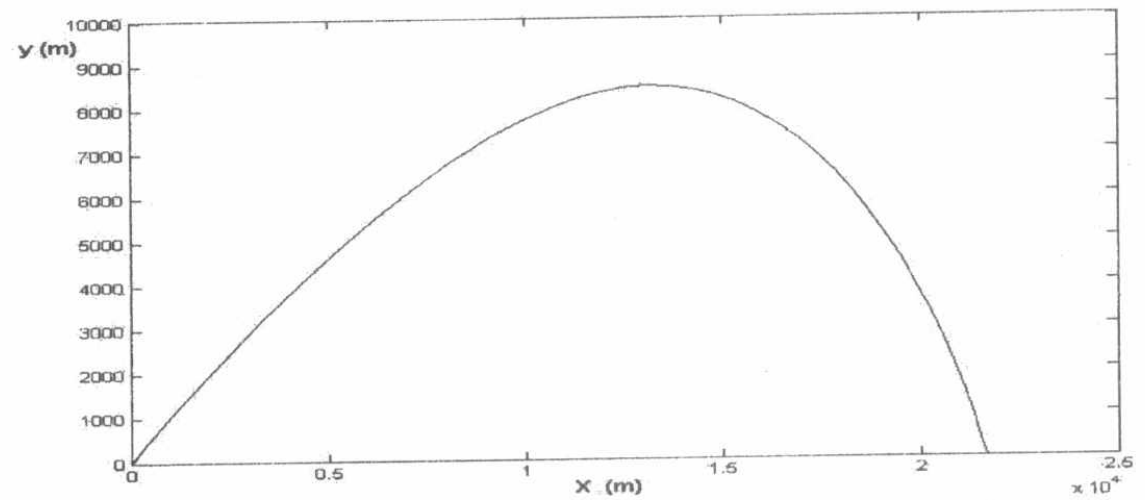

Fig. 7 Trajectory plot of the rocket assisted projectile of

(a ratio of propellant to projectile mass $=3 \%$, rocket time of function $=1.0 \mathrm{~s}$ delay time $=2.0 \mathrm{~s}$, firing angle $=47^{\circ}$ )

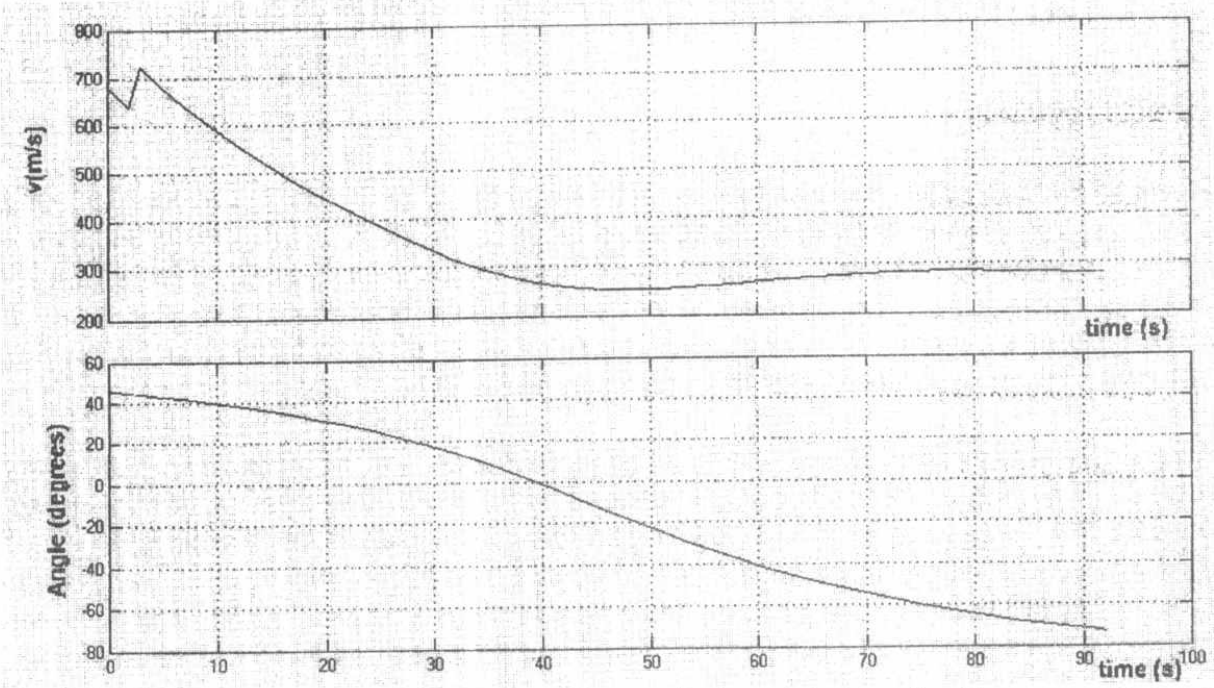

Fig. 8 Velocity and inclination angle of the rocket assisted projectile versus time of flight

(a ratio of propellant to total projectile mass $=3 \%$, rocket time of function $=1.0 \mathrm{~s}$, delay time $=2.0 \mathrm{~s}$, and firing angle $=47^{\circ}$ )

\section{4 Firing Angle of Maximum Range}

The programmed code is used to compute the firing angle of maximum range of different mass ratios $(0: 0.1)$ for different times of function $(0.5,1,2$ and 5$) \mathrm{s}$ and 
different delay times $(0,1,2,5,10$ and 20$)$ s. obtained data, Fig. 9 shows that the firing angle of maximum range depends only on the mass ratio, neither on the delay time nor the time of function.

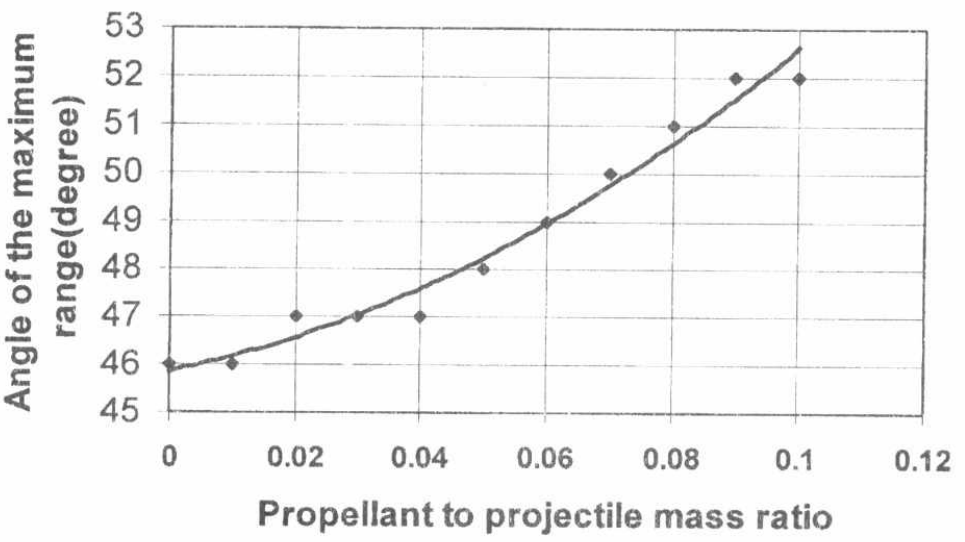

Fig. 9 The firing angle of maximum range depends on the mass ratio

\section{CONCLUSION}

A versatile computer program is coded using SIMULINK solver module to compute the trajectory elements of the rocket assisted projectiles. The program can be used to study the influence of the different design factors on the range extension of the rocket assisted projectiles. The program is considered as a useful tool to extend the range of any other projectile by using an assisting rocket, provided that the mass, muzzle velocity and drag coefficient of the projectile are known.

The study shows that, the range can be significantly extended by using an assisting rocket. The higher the ratios of the propellant mass to the total projectile mass the greater the range extension. The firing angle of the maximum range is higher than that of the original projectile and is a function of the ratio of the propellant mass to the total projectile mass. The shorter the delay time of starting the assisting rocket the greater the range extension. The influence of the time of function of the assisting rocket on the range is very small.

\section{REFERENCES}

1. Kamal, Osama I., Abdalla, Hamed and Sarhan, Ahmed M., "Numerical Solution Of Laminar Separated Flow Over The base Of A Cylinder," 7th Applied Mechanics and Mechanical Engineering Conference, pp.485-499, 28-30 May, 1996.

2. Yakot, H. and Abdel-kader, M.S. (1990)" Range Extension Via Base Bleeding," $4^{\text {th }}$ Applied Mechanial Engineering Conference, M.T.C., Cairo. 
3. Yakot, H. and Abdel-kader, M.S. (1991)" Assessment of ERFB-BB Projectile," $4^{\text {th }}$ Aeronautical Sciences \&Aviation Technology Conference, M.T.C., Cairo.

4. Anderson, K., "Different Methods to Reach Long Range $\geq 65 \mathrm{~km}$ for Future $155 \mathrm{~mm}$ Artillery Systems, Possibilities \& Limitations, "17 th International Symposium on Ballistics, South Africa, vol. I, pp. 149-156, 1998.

5. Karsten, P. A., "Long Range Artillery: The Next Generation," $17^{\text {th }}$ International Symposium on Ballistics, South Africa, vol. I, pp. 133-141, 1998.

6. NASA, Glenn Research Center, Earth Atmosphere Model, Metric Units,

7. Sarhan, Ahmed M. and Nafea, Aly, "Experimental Determimation Of Projectile Drag Coefficient Using Pulsed Radar Doppler Technique, "Ninth 18, 2000. 